
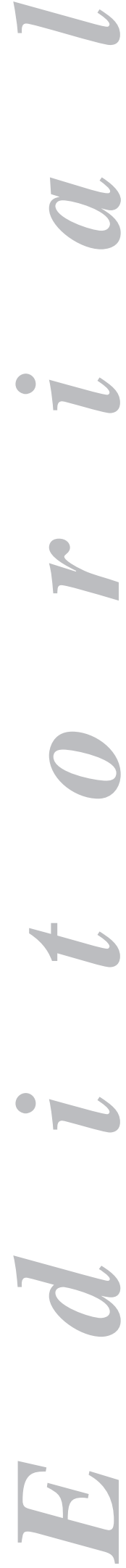

\section{Establishing prognosis in alcoholic hepatitis}

Alcoholic hepatitis (AH) is a clinical syndrome characterized by the development of jaundice and/or decompensation signs in a patient with excessive, long-term use of alcohol. AH may develop at any time during alcoholic liver disease, but most cases involve patients with advanced fibrosis or cirrhosis (1). The clinical picture is associated with typical histological lesions including steatosis, inflammatory infiltration mainly by polymorphonuclear cells, cell ballooning, and Mallory-Denk hyaline bodies. The presence of megamitochondria and -particularly in patients with bacterial infection- bilirubinostasis is also common.

AH remains a relevant cause of morbidity and mortality, with immediate mortality ranging from $15 \%$ to $50 \%$ of cases according to severity. A joint analysis of the various controlled studies shows that $34 \%$ of patients who did not receive corticosteroids died within the first 28 days (2).

AH treatment has remained unchanged for the last 3 decades, and is still based on alcohol withdrawal, correction of nutritional deficiencies, and use of corticosteroids in doses of $40 \mathrm{mg} /$ day for 4 weeks. Alternatively, the use of pentoxifylline has been suggested for patients in whom corticoids are contraindicated (3). However, this treatment is far from being satisfactory since a significant proportion of patients have no response and, additionally, corticoids are not exempt from complications, particularly infection.

In the last few years notable advances have occurred in the understanding of molecular mechanisms associated with $\mathrm{AH}$ pathogenesis, which has led to the identification of potential therapy targets (4). In order to appropriately assess the efficacy of novel therapies a number of criteria must be met, including: Early suspicion of the diagnosis of AH in an alcoholic patient with a suddenly decompensated liver disease, establishing severity and death risk, and histological confirmation of diagnosis when possible.

In 1978 Maddrey et al. described their discriminant function in a controlled study to assess the effectiveness of corticoids in the management of AH (5). This index is based on bilirubin levels and prothrombin time, two lab parameters that have shown prognostic value in all studies. This index was modified in 1989 by using the difference in prothrombin time (in seconds) between a patient and a control subject (6). Patients not on corticoids with an index equal to or higher than 32 have a 28-day survival of $68 \%$, and clinical guidelines recommend that they be treated. The benefits of this index include simplicity and proven efficacy in multiple studies. While mortality is present for patients with an index below 32, a risk-benefit analysis advises against systematically treating these patients.

Other indices that were suggested more recently include MELD (7), Glasgow Index (8), and ABIC (9). All of them include, besides bilirubin and prothrombin, a renal function parameter - creatinine or BUN in ABIC, and age and WBC count in Glasgow Index (Table I). Portal pressure gradient is also associated with hospital mortality 
for patients with serious $\mathrm{AH}$, but this technique is only used in some specialized centers (10). Initial studies regarding these indices show their good predictive value for mortality at 30 and 90 days. However, they have been insufficiently validated in independent cohorts, and time points are also inadequately established in some cases. Another limitation for most of these indices is their stratification of patients in only two categories -- severe and not severe. Similarly, they focus on immediate mortality. ABIC, in an attempt at bypassing these limitations, allows a classification of patients into three severity groups and has sustained prognostic value at 6 and 12 months.

In the article reported in this issue of Revista Española de Enfermedades Digestivas, Bargalló et al. (11) discuss the predictive factors for mortality in a series of 61 patients with severe AH that was collected along 8 years. Mortality at 30 days (16.9\%) was similar to that seen in other series. This study compares the various prognostic indices and notes that MELD is slightly superior; also, regarding long-term survival, only MELD was predictive at 7 days. This is an interesting contribution, albeit with the limitations inherent to a retrospective study in a relatively small number of patients.

The clinical utility of the various predictive indices has been compared by several studies with conflicting results, also due to the limitations seen in the present study. These are retrospective studies in relatively small series of patients that compare heterogeneous patient samples because of differing patient proportions with histological confirmation, patient percentages with $\mathrm{AH}$-associated cirrhosis, inclusion and exclusion criteria, patient proportions receiving treatment with corticoids, and periods of time for survival assessment (12). In a prospective study in a cohort of 332 patients with biopsy-confirmed AH both MELD and ABIC showed good predictive values with an area under the ROC curve above 0.7 (13). In the original study, the prognostic value of ABIC was somewhat superior to MELD, in contrast with the study by Bargalló et al., but differences are scarcely significant.

Immediate mortality from AH is closely related to early liver function changes. In this respect, a MELD score increase by 2 or more points within the first week in hospital is associated with a significantly higher mortality (14). The Lille Index establishes prognosis based on response to treatment with prednisolone and incorporates bilirubin levels after one week on treatment (15). More recently two new cut-offs could be added to the Lille Index to categorize patients as responders, partial responders or non-responders, which allow a prediction of survival after two months (2). From all this it is no wonder that parameters with the best prognostic values were obtained at one week in the study by Bargalló et al. This has the disadvantage of excluding patients dying within the first 7 days, who represent a relevant proportion of all those

Table I. Prognostic indices for alcoholic hepatitis

\begin{tabular}{|c|c|c|c|}
\hline $\begin{array}{l}\text { Modified Maddrey's } \\
\text { DF: } 4.6 \text { x [patient p }\end{array}$ & $\begin{array}{l}\text { tion } \\
\text { - control }\end{array}$ & time (secor & $\mathrm{n}(\mathrm{mg} / \mathrm{dL})$ \\
\hline $\begin{array}{l}\text { MELD: } \\
3.8 \times \text { loge(bilirubin }\end{array}$ & loge(INF & (creatinine & \\
\hline Glasgow index: & 1 & 2 & 3 \\
\hline Age & $<50$ & $>50$ & - \\
\hline Leukocytes (109xL) & $<15$ & $>15$ & - \\
\hline Urea (mmol/L) & $<5$ & $>5$ & - \\
\hline Prothrombin (ratio) & $<1.5$ & $1.5-2$ & $>2$ \\
\hline Bilirubin $(\mu \mathrm{mol} / \mathrm{l})$ & $<125$ & $125-250$ & $>250$ \\
\hline
\end{tabular}


who pass away during hospitalization. Other factors affecting immediate mortality from AH include bacterial infection (16), as seen in the study by Bargalló et al., as well as impaired renal function (17) and systemic inflammatory response syndrome (18), although none of these parameters has been included in prognostic indices.

Three factors greatly condition the long-term prognosis of AH that are scarcely emphasized in the paper by Bargalló et al. These include alcohol withdrawal, presence of cirrhosis, and response to steroid therapy. Withdrawal is the single most important predictor in AH once the initial stage is over, and is also essential when considering transplantation (19). Therefore, dependency therapy should be initiated during hospitalization to be followed by stringent monitoring for at least two years after discharge. In the present article a clear tendency may be seen towards better survival with withdrawal, although significance was not reached given the number of included patients. The relatively low percentage of patients with cirrhosis as compared to other series of patients with similar characteristics is somewhat surprising. Cirrhosis may have been underdiagnosed as biopsies were available for only $53 \%$ of patients.

The authors consider, as many others do, that biopsy is not essential for the diagnosis of AH. However, the latest EASL guidelines indicate that, while AH may be suspected from clinical and laboratory criteria, a definitive diagnosis requires a biopsy procedure (3). In fact, biopsy cannot confirm a suspected diagnosis in around $20 \%$ of patients (20). Indeed, the fact that biopsy is to be performed via the transjugular route precludes its use in many centers, but biopsy obtention from the highest number of patients possible should become a priority goal. Biopsy is key for the inclusion of patients in a therapeutic study. In addition, the information provided by biopsy may also be of predictive value. Recently, a multicenter study was performed to develop a histological index to predict short-term survival in patients with AH. This index (the Alcoholic Hepatitis Histological Score, AHHS) comprises four parameters independently associated with survival: Fibrosis extent, infiltration with polymorphonuclear cells, bilirubinostasis, and presence of megamitochondria (21). By using these 4 parameters in combination patients with a low, intermediate or high risk of mortality at 90 days can be stratified.

An ideal prognostic index should be simple, accessible for all centers, objective, and validated in external cohorts from populations of various origins. For the time being, the Maddrey and Lille indices remain the most widely used scores for treatment onset and response monitoring, respectively. Indices posited more recently have also proven useful for therapy indication, and ABIC among them has the advantage of patient stratification into three groups, which eases both treatment indication and outcome interpretation. Although these indices are good predictors of mortality, the combination of a clinical index with a histological index may contribute to a better categorization of patients with alcoholic hepatitis according to severity.

Juan Caballeria

Liver Unit. Hospital Clínic. CIBERehd. IDIBAPS. Barcelona, Spain

\section{REFERENCES}

1. Lucey MR, Mathurin P, Morgan TR. Alcoholic hepatitis. N Engl J Med 2009;360:2758-69.

2. Mathurin P, O'Grady J, Carithers RL, Philips M, Louvet A, Mendenhall CL, et al. Corticosteroids improve short-term survival in patients with severe alcoholic hepatitis. Meta-analysis of individual patient data. Gut 2011;60:255-60. 
3. EASL Clinical Practical Guidelines: Management of alcoholic liver disease. J Hepatol 2012;57:339-420.

4. Gao B, Bataller R. Alcoholic liver disease: Pathogenesis and new therapeutic targets. Gastroenterology 2011;141:1572-85.

5. Maddrey WC, Boitnott K, Bedine MS, Weber FL, Mezey E, White RL. Corticosteroid therapy of alcoholic hepatitis. Gastroenterology 1978;75:193-9.

6. Carithers RL Jr, Herlong HF, Diehl AM, Shaw EW, Combes B, Fallon HJ, et al. Methylprednisolone therapy in patients with severe alcoholic hepatitis. A randomized multicenter trial. Ann Intern Med 1989;110:685-90.

7. Dunn W, Jamil LH, Brown LS, Wiesner RH, Kim WR, Menon KVN, et al. Meld accurately predicts mortality in patients with alcoholic hepatitis. Hepatology 2005;41:353-8.

8. Forrest EH, Evans CD, Stewart S, Phillips M, Oo YH, McAvoy NC, et al. Analysis of factors predictive of mortality in alcoholic hepatitis and derivation and validation of the Glasgow alcoholic hepatitis score. Gut 2005;1174-9.

9. Domínguez M, Rincón D, Abraldes JG, Miquel M, Colmenero J, Bellot P, et al. A new scoring system for prognostic stratification of patients with alcoholic hepatitis. Am J Gastroenterol 2008;103:2745-56.

10. Rincon D, Lo Iacono O, Ripoll C, Gómez-Camarero J, Salcedo M, Catalina MV, et al. Prognostic value of hepatic venous pressure gradient for in-hospital mortality of patients with severe acute alcoholic hepatitis. Aliment Pharmacol Ther 2007;25:841-8.

11. Bargalló-García A, Serra-Matamala I, Marin-Fernánez I, Masnou-Ridaura H, Leal-Valdivieso C, Marcos-Neira $\mathrm{P}$, et al. Prognostic factors associated with mortality in patients with severe alcoholic hepatitis. Rev Esp Enferm Dig 2013;105:513-20.

12. Singal AK, Shah VH. Alcoholic hepatitis: Prognostic models and treatment. Gastroenterol Clin N Am 2011;40:611-39.

13. Louvet AA, Wartel F, O'Grady JG, et al. Prognostic scores are efficient in patients with severe alcoholic hepatitis treated with corticosteroids: Comparison of available models. Hepatology 2010;52(Supl. 1):1108-1109A.

14. Srikureja W, Kyulo NL, Runyon BA, Hu KQ. Meld is a better prognostic model than Child-Turcotte-Pugh score or discriminant function score in patients with alcoholic hepatitis. J Hepatol 2005;42:700-6.

15. Louvet A, Naveau S, Abdelnour M, Ramond MJ, Diaz E, Fartoux L, et al. The Lille model: A new tool for therapeutic strategy in patients with severe alcoholic hepatitis treated with steroids. Hepatology 2007;45:1348-54.

16. Louvet A, Wartel F, Castel H, Dharancy S, Hollebecque A, Canva-Delcambre V, et al. Prospective screening of infection in patients with severe alcoholic hepatitis treated with steroids: Early response to therapy is the key factor. Gastroenterology 2009;137:541-8.

17. Altamirano J, Fagundes C, Domínguez M, García E, Michelena J, Cárdenas A, et al. Acute kidney injury is an early predictor of mortality for patients with alcoholic hepatitis. Clin Gastroenterol Hepatol 2012;10:65-71.

18. Michelena J, Altamirano J, Arroyo V, Caballeria J, Bataller R. Alcoholic hepatitis-associated systemic inflammatory response syndrome at admission is a major determinant of multiple organ dysfunction and mortality. Hepatology 2012;56:983A.

19. Pessione F, Ramond MJ, Peters L, Pham BN, Batel P, Rueff B, et al. Five-year survival predictive factors in patients with excessive alcohol intake and cirrhosis. Effect of alcoholic hepatitis, smoking and abstinence. Liver Int 2003;23:45-53.

20. Mookerjee RP, Lackner C, Stauber R, Stadlbauer V, Deheragoda M, Aigelsreiter A, et al. The role of liver biopsy in the diagnosis and prognosis of patients with acute deterioration of alcoholic cirrhosis. J Hepatol 2011;55:1103-11.

21. Altamirano J, Miquel R, Katoonizadeh A, Duarte-Rojo A, Smirk TC, Michelena J, et al. Development and validation of a novel histological classification with prognostic value for alcoholic hepatitis. Hepatology 2011;54 (Supl.):968A 